\title{
X-ray tests of a Pixel Array Detector for coherent $x$-ray imaging at the Linac Coherent Light Source
}

\author{
L.J. Koerner, ${ }^{a}$ H.T. Philipp, ${ }^{a}$ M.S. Hromalik, ${ }^{a}$ M.W. Tate ${ }^{a}$ and S.M. Gruner ${ }^{a, b, 1}$ \\ ${ }^{a}$ Laboratory of Atomic and Solid State Physics, Physics Department, Cornell University, \\ Ithaca, 14853 New York, U.S.A. \\ ${ }^{b}$ Cornell High Energy Synchrotron Source (CHESS), Cornell University, \\ Ithaca, 14853 New York, U.S.A.
}

E-mail: smg26@cornell.edu

\begin{abstract}
Test results are presented of a pixel array detector (PAD) developed for $\mathrm{x}$-ray imaging at the Stanford Linear Coherent Light Source (LCLS). The basic module of the PAD consists of two bump-bonded chips: a reverse-biased silicon diode chip of $185 \times 194$ pixels, each of which is coupled by bump-bonds to a charge integrating CMOS ASIC with digitization in each pixel. The LCLS experiment requires a high signal-to-noise ratio for detection of single $8 \mathrm{keV} \mathrm{x}$-rays, a pixel full-well exceeding $1,0008 \mathrm{keV}$ x-rays, a frame-rate of $120 \mathrm{~Hz}$, and the ability to handle the arrival of thousands of $\mathrm{x}$-rays per pixel in tens of femtoseconds. Measurements have verified a pixel full-well value of 2,700 $8 \mathrm{keV}$ x-rays. Single $8 \mathrm{keV}$ photon detection has been shown with a signal-to-noise ratio of $>6$. Line-spread response measurements confirmed charge spreading to be limited to nearest neighbor pixels. Modules still functioned after dosages up to $75 \mathrm{Mrad}(\mathrm{Si})$ at the detector face. Work is proceeding to incorporate an array of modules into a large-area detector.
\end{abstract}

KEYWORDS: X-ray detectors; Pixelated detectors and associated VLSI electronics; X-ray diffraction detectors

\footnotetext{
${ }^{1}$ Corresponding author.
} 


\section{Contents}

1 Introduction 1

1.1 Coherent imaging experiment at the LCLS 1

1.2 Detector characteristics 2

2 Testing results $\quad 3$

2.1 Methods 3

2.2 Pixel saturation, linearity and noise 3

2.3 Spatial response 4

2.4 Radiation robustness 4

3 Results summary 6

4 Conclusions 6

\section{Introduction}

\subsection{Coherent imaging experiment at the LCLS}

The Linac Coherent Light Source (LCLS), a free-electron laser (FEL), is being developed at the Stanford Linear Accelerator Center (SLAC). The laser will produce extremely bright, temporally short $\mathrm{x}$-ray pulses at a wavelength of $1.5 \AA(8 \mathrm{keV})$ [1]. One experiment planned for this FEL is single-shot coherent imaging of single biological molecules or particles inserted one-by-one into the pulsed x-ray beam [2]. Upon x-ray exposure, the particle ejects photoelectrons, becomes positively charged, and then Coulomb explodes within femtoseconds. Ideally, the laser would provide an x-ray pulse of temporal width smaller than the time for Coulomb explosion. In this case, the x-rays would have scattered off a native structure before the particle structure changes due to the explosion [3,4]. Since only a relatively small number of x-rays are scattered by a single particle, the complete data set must be assembled by many repetitions of the experiment. A successful development of this technique would provide the opportunity to determine the atomic level structure of biological particles without crystallization. The scattering intensity profile will drop strongly with scattering angle as $1 / \theta^{4}$, where $\theta$ is the scattering angle [2]. Thousands of $\mathrm{x}$-rays per pixel are anticipated at the central region of the detector while an average of less than one photon per pixel is expected in the wide-angle regions. The LCLS pulses at $120 \mathrm{~Hz}$, so new particles may be injected into the beam every $8.3 \mathrm{~ms}$. 


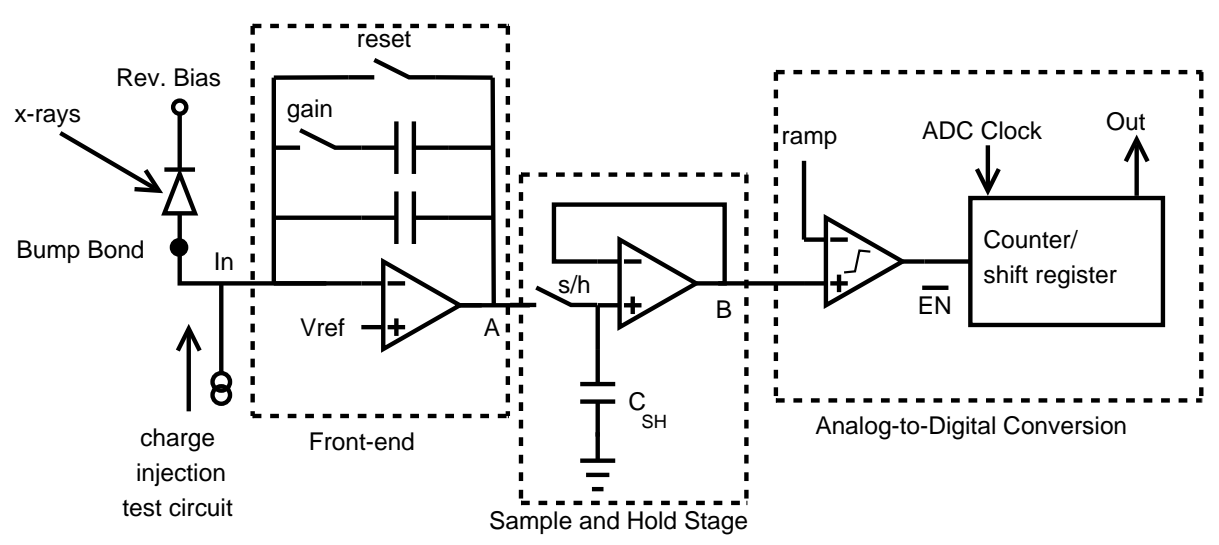

Figure 1: Pixel schematic displaying, from left to right, the front-end capacitive transimpedance amplifier with 1-bit programmable gain selection, a low-bandwidth sample-and-hold stage, and a single-slope analogto-digital converter. The pixel measures $110 \mu \mathrm{m} \times 110 \mu \mathrm{m}$.

\subsection{Detector characteristics}

The experiment imposes several demands upon the detector design: a large signal-to-noise ratio at low illumination levels, a pixel full-well greater than $1,0008 \mathrm{keV}$-rays, and a frame rate of $120 \mathrm{~Hz}$. To meet these requirements a pixel array detector (PAD), consisting of a high-resistivity silicon detector layer bump-bonded to a readout application-specific integrated circuit (ASIC), is being developed. PADs couple the low conversion noise and excellent spatial response of direct $\mathrm{x}$-ray conversion in silicon with the flexibility and speed of a readout ASIC designed in standard CMOS. The detector layer is fabricated by SINTEF (Trondheim, Norway) using high-resistivity $500 \mu \mathrm{m}$ thick n-type silicon. $\mathrm{p}^{+}$pixel implants are formed on the bump-bonded side and an aluminized $\mathrm{n}^{+}$ohmic contact is applied on the $\mathrm{x}$-ray incident side for the application of an $\approx 200 \mathrm{~V}$ bias to fully deplete the thickness of the sensor. The readout ASIC is fabricated in TSMC $0.25 \mu \mathrm{m}$ feature-size CMOS mixed-mode process through the MOSIS service (Marina del Rey, California, U.S.A.). Detector and ASIC chips were bump-bonded using tin-lead solder by RTI (Research Triangle Park, NC, U.S.A.) [5]. The pixel circuitry consists of a low-noise charge integrating front-end with per-pixel configurable two-level gain followed by an in-pixel single-slope 14-bit analog-todigital converter (A/D) [6,7] (refer to figure 1). The in-pixel digital numbers are read-out via pixel addressing using bit-passing registers and an 8-bit wide bus at a rate of $25 \mathrm{MHz}$, which results in a full-frame read-out time of $3.1 \mathrm{~ms}$.

A single readout ASIC measures $21.35 \times 21.30 \mathrm{~mm}$ with a $185 \times 194$ array of $110 \mu \mathrm{m} \times 110 \mu \mathrm{m}$ pixels. Test results presented below are on single-module units, i.e., a single detector chip bumpbonded to a single readout ASIC. The final detector configuration will be built from modules of two readout ASICs bump-bonded to one detector array of $185 \times 388$ pixels with two rows of elongated $(275 \mu \mathrm{m})$ pixels to bridge the space required to abut two ASIC chips. Eight of these modules will be tiled around a hole for passage of the direct $\mathrm{x}$-ray beam, to create a detector unit of $758 \times 758$ pixels. 

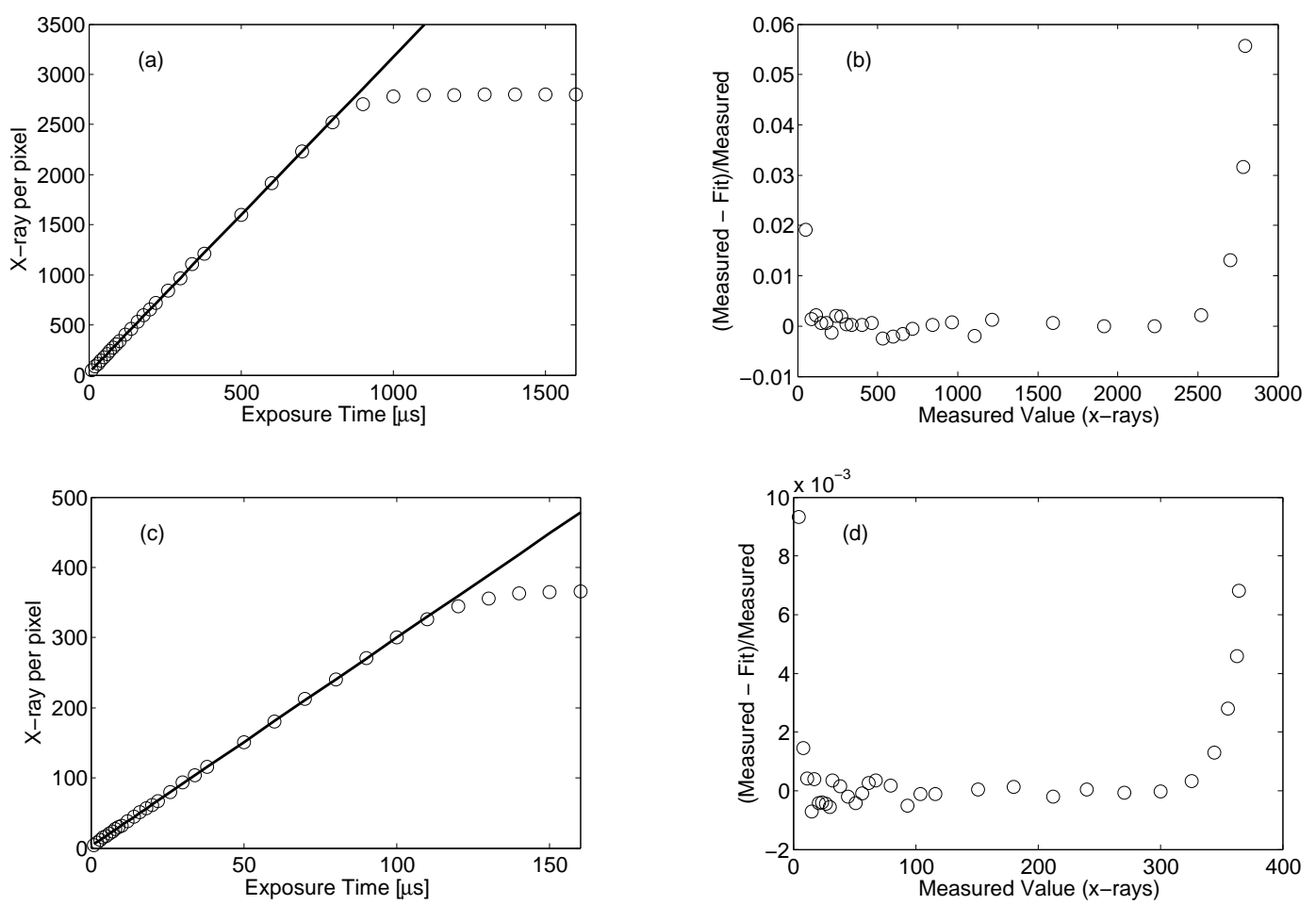

Figure 2: Measured x-rays versus exposure time (a, c) and fractional residuals from a linear fit (b, d) for low-gain (a, b) and high-gain (c, d).

\section{Testing results}

\subsection{Methods}

Bump-bonded modules were wire-bonded to a support card and mounted in a vacuum cryostat with a thermoelectrically regulated copper cold-finger and an x-ray transparent, light-tight aluminized mylar window. Detector control and data acquisition was accomplished with a Xilinx Virtex4 FPGA on a PCIeSYS100FX development board from PLDA (San Jose, CA) [8]. A rotating copper anode source (FR571; Enraf Nonius, Bohemia, NY, U.S.A.) collimated by confocal multi-layer mirrors (CMF15-165Cu8, Osmic, Troy, NY, U.S.A.) was used for spot response and radiation damage testing. A $50 \mathrm{~W}$ TFS-6050 copper microfocus x-ray tube powered by a TCM-500M supply (Trufocus, Watsonville, CA, U.S.A.) was used for flat-field testing.

\subsection{Pixel saturation, linearity and noise}

Detector linearity and pixel saturation value were tested by varying the exposure-time while illuminating with an $\mathrm{x}$-ray spot of $\approx 1 \mathrm{~mm} \times 1 \mathrm{~mm}$. The average per-pixel $\mathrm{x}$-ray value versus exposuretime are shown for both high and low-gain configurations in figures $2 \mathrm{a}$ and $2 \mathrm{c}$. Fractional residuals from a linear fit versus measured illumination are shown in figures $2 \mathrm{~b}$ and $2 \mathrm{~d}$. The pixel saturation level (defined as the point of $2 \%$ non-linearity) was measured to be $2,7008 \mathrm{keV} \mathrm{x}$-rays $\left(5.9 \times 10^{6}\right.$ electrons $)$ in low-gain and $3508 \mathrm{keV}$ x-rays $\left(0.75 \times 10^{6}\right.$ electrons $)$ in high-gain.

Single-photon sensitivity was confirmed using monochromatic $8 \mathrm{keV}$ radiation incident on the detector through a $25 \mu \mathrm{m}$ pin-hole centered on a single pixel to eliminate charge sharing. Figure 3 shows a histogram of single-pixel values acquired in high-gain mode using three expo- 


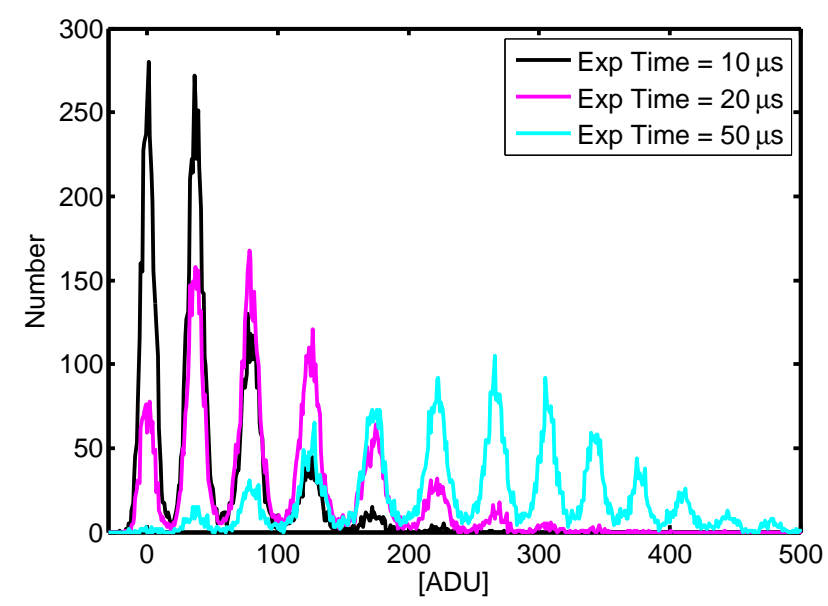

Figure 3: Single pixel response histogram acquired in high-gain mode using $8 \mathrm{keV}$ radiation incident on the detector through a $25 \mu \mathrm{m}$ diameter pin-hole centered on a pixel. The leftmost peak corresponds to zero $\mathrm{x}$-rays per exposure, the next to one x-ray per exposure, and so on. ADU is the background subtracted measured digital value and Number is the number of occurrences. Exposure times are differentiated by color and indicated in the legend.

sure times, with 10,000 frames acquired per exposure time. The signal-to-noise is sufficiently high to enable counting of individual photons in the integrated signal as evidenced by discrete peaks in figure 3. The dynamic range is calculated from the noise and linearity measurements as $\mathrm{DR}=\frac{F W_{\mathrm{LG}}}{\sigma_{\mathrm{HG}}}=18,000=85 \mathrm{~dB}$, where $F W_{\mathrm{LG}}$ is the pixel saturation value in low-gain and $\sigma_{\mathrm{HG}}$ is the rms read-noise in high-gain. Read-noise measurements are summarized in table 1.

\subsection{Spatial response}

To study the spatial response, a uniform x-ray illumination field was occluded using a tungsten knife-edge and the detector was translated by a micro-positioning stage (ILS, Newport, Irvine, CA, U.S.A.). The response from many pixels was aligned to form the pixel edge-spread response shown in figure 4a. The line-spread response is calculated as the spatial derivative of the edge-spread response (using a $5 \mu \mathrm{m}$ window) and is shown in figure $4 \mathrm{~b}$. The normalized intensity fell to $20 \%$ and $5 \%$ at a distance of $8 \mu \mathrm{m}$ and $14 \mu \mathrm{m}$ from the pixel boundary, respectively. This shows that charge sharing only occurs when x-rays are incident in a zone about $20 \mu \mathrm{m}$ from the edge of the pixel. An example x-radiograph of a U.S. one dollar bill, illustrating the sharp spatial response of the detector, is shown in figure 5. X-ray absorption contrast is due to the green pigment in the dollar.

\subsection{Radiation robustness}

An ASIC without a matching Si detector layer was dosed at a rate of $193 \mathrm{rad}(\mathrm{Si}) / \mathrm{sec}$ with $8 \mathrm{keV}$ x-rays while held at $12^{\circ} \mathrm{C}$ and electrically biased. An array of spots of different levels of accumulated dose, up to $400 \mathrm{krad}(\mathrm{Si})$, was distributed onto the chip. Failure of pixel functionality occurred at $\simeq 140 \mathrm{krad}$. The failures were localized to the comparator element in the single-slope A/D and are hypothesized to be the result of radiation-induced parasitic field-oxide transistors. Following a 43 day interval, during which the chip was unbiased and at room temperature, pixel functionality returned at all dose levels (up to $400 \mathrm{krad}$ ). Residual effects were seen in slight shifts of the 

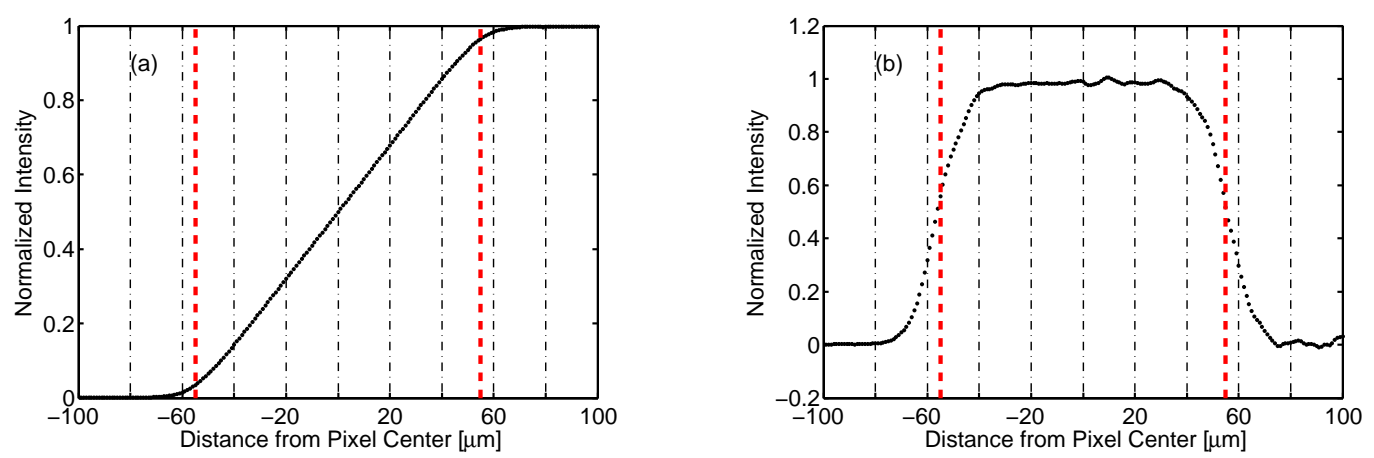

Figure 4: (a) Composite pixel edge-spread response and (b) line-spread response. Dashed red-lines indicate pixel boundaries.

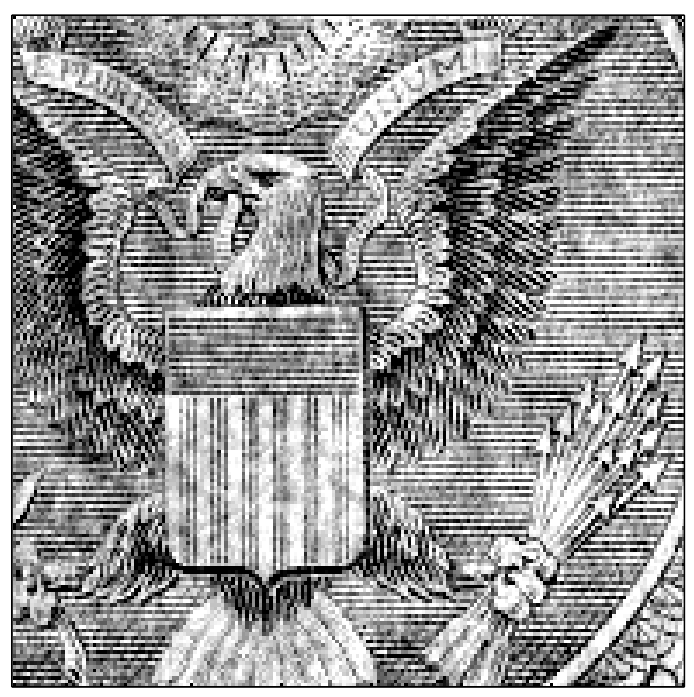

Figure 5: An $x$-radiograph of a portion of a U.S. one dollar bill taken using $8 \mathrm{keV}$ radiation. There is $10 \%$ contrast from dark to light in the image.

background levels and an increase in the noise of the damaged pixels. An additional 21 hour $56^{\circ} \mathrm{C}$ electrically biased annealing period to the same dosed chips removed all signs of radiation damage.

A hybridized module of ASIC and detector layer was dosed up to $75 \mathrm{Mrad}(\mathrm{Si})$ at the detector front face with $8 \mathrm{keV}$ x-rays. The detector pixels continued to function to specification as evidenced by the acquisition of a single pixel response histogram similar to that shown in figure 3 . The $\mathrm{x}$-ray absorption of the detector diode implies an accumulated dose of $64 \mathrm{krad}(\mathrm{Si})$ at the ASIC, ignoring absorption from the tin-lead bump-bonds. After dosing to $75 \mathrm{Mrad}$, the detector layer leakage currents, measured at $-14{ }^{\circ} \mathrm{C}$ and a bias of $185 \mathrm{~V}$, increased from $44 \mathrm{fA} /$ pixel to $124 \mathrm{fA} /$ pixel. I-V scans of damaged and undamaged detector portions are shown in figure 6a. The activation energy, $A$, relates to the leakage current, $I$, and temperature, $T$, as $I \propto e^{-A / k T}$ and is plotted versus reverse bias in figure $6 \mathrm{~b}$. The origin of the leakage current increase is hypothesized to be surface states at the $\mathrm{SiO}_{2}-\mathrm{Si}$ interface, which are known to have a broad density of states as a function of energy con- 

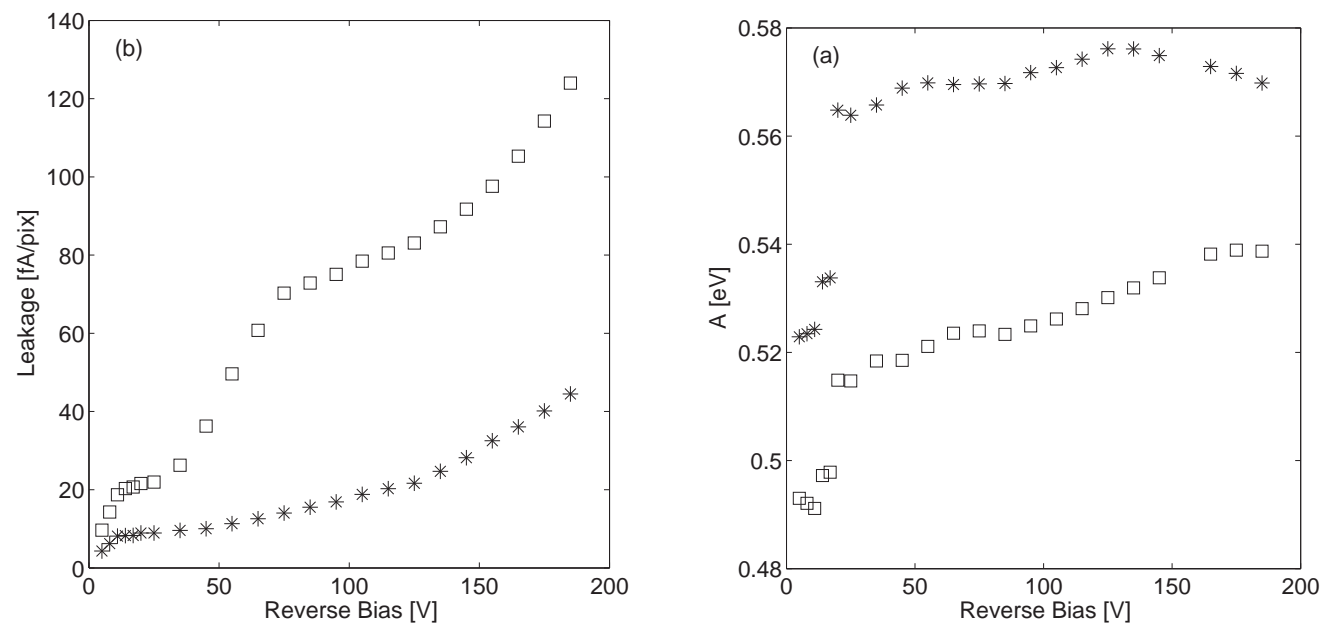

Figure 6: (a) Detector layer I-V scans for un-dosed portions (*) and portions dosed to $75 \mathrm{Mrad}(\square)$ acquired at $-14^{\circ} \mathrm{C}$. (b) From I-V scans at nine different temperatures the activation energy of the leakage current is extracted and plotted for dosed and un-dosed portions versus bias.

Table 1: Parameters of the prototype PAD. X-ray values refer to $8 \mathrm{keV}$ x-rays. HG indicates a measurement result with the detector front-end configured in high-gain mode ( $75 \mathrm{fF}$ integration capacitance). LG indicates a measurement result with the detector front-end configured in low-gain mode ( $556 \mathrm{fF}$ integration capacitance).

\begin{tabular}{|ll|}
\hline Pixel Size & $110 \mu \mathrm{m} \times 110 \mu \mathrm{m}$ \\
Array Size & $185 \times 194$ pixels \\
Frame rate & $120 \mathrm{~Hz}$ \\
Read-Noise (RMS) & $350 \mathrm{e}^{-}(\mathrm{HG}) ; 1000 \mathrm{e}^{-}(\mathrm{LG})$ \\
& $0.16 \mathrm{x}$-rays $(\mathrm{HG}) ; 0.46 \mathrm{x}$-rays $(\mathrm{LG})$ \\
& $350 \mathrm{x}$-rays $(\mathrm{HG}) ; 2,700 \mathrm{x}-$ rays $(\mathrm{LG})$ \\
Full-well & $0.97(8 \mathrm{keV}) ; 0.89(12 \mathrm{keV}) ; 0.48(18 \mathrm{keV})$ \\
Quantum Efficiency $^{a}$ & $40 \mathrm{fA} / \mathrm{pix}, 0.33 \mathrm{nA} / \mathrm{cm}^{2}\left(-14{ }^{\circ} \mathrm{C}\right) ; 700 \mathrm{fA} / \mathrm{pix}, 5.8 \mathrm{nA} / \mathrm{cm}^{2}\left(18^{\circ} \mathrm{C}\right)$ \\
Dark Current & $0.00011 \mathrm{x}$-rays $/ \mu \mathrm{s} / \mathrm{pixel} ; 0.002 \mathrm{x}$-rays $/ \mu \mathrm{s} /$ pixel \\
Bump-Bond Yield $^{b}$ & $0.99987 ; 0.99891$ \\
\hline
\end{tabular}

${ }^{a}$ Calculated values assuming charge from x-rays converting in the undepleted $\mathrm{n}^{+}$ohmic contact is not transported to the pixel electrodes (conservative approximation as charge may escape via diffusion [11]).

${ }^{b}$ Median and mean bump-yield, respectively, of 16 assemblies tested. All but one module (0.987) measured bump-yields $>0.9987$.

centrated near mid-gap and form when capture of x-ray induced holes breaks Si-H bonds $[9,10]$.

\section{Results summary}

\section{Conclusions}

Prototype PADs have been tested with x-rays and demonstrated to achieve the required noise, spatial response, saturation values, and radiation robustness for the experiment. Measurements confirmed that the point illumination spread was limited to the nearest neighbor, displayed clear 
quantized photon detection, and showed linear response to the desired saturation value. In addition, radiation robustness was evaluated and bump-bonding yield was quantified (results are shown in table 1). In future work, single chips will be slightly modified for better power supply distribution across the chip. These will be incorporated into modules that can be abutted side-byside and support electronics will be upgraded to build a large-area detector $(758 \times 758$ pixels, $83.8 \mathrm{~mm} \times 83.8 \mathrm{~mm})$.

\section{Acknowledgments}

The authors acknowledge Martin Novak for expert machining of the detector cryostat, Daniel Schuette for useful discussions, and Nozomi Ando for editorial assistance. The project is funded through the Stanford Linear Accelerator Center (SLAC) as part of a DOE BES project to develop the Linac Coherent Light Source (LCLS) facilities. Detector development at Cornell is also supported by DOE-BER grant DE-FG02-97ER62443.

\section{References}

[1] R. Tatchyn et al., Research and development toward a 4.5-1.5 ^ linac coherent light source (LCLS) at SLAC, Nucl. Instrum. Meth. A 375 (1996) 274.

[2] K.J. Gaffney and H.N. Chapman, Imaging atomic structure and dynamics with ultrafast x-ray scattering, Science 316 (2007) 1444.

[3] G. Huldt, A. Szöke and J. Hajdu, Diffraction imaging of single particles and biomolecules, J. Struct. Biol. 144 (2003) 219.

[4] R. Neutze, R. Wouts, D. van der Spoel, E. Weckert and J. Hadju, Potential for biomolecular imaging with femtosecond X-ray pulses, Nature 406 (2000) 752.

[5] A. Huffman, R. LaBennett, S. Bonafede and C. Statler, Fine-pitch wafer bumping and assembly for high density detector systems, IEEE Nucl. Sci. Symp. Conf. Rec. 5 (2003) 3522.

[6] S. Kleinfelder, S. Lim, X. Liu and A.E. Gamal, A 10000 frames/s CMOS digital pixel sensor, IEEE J. Solid State Circ. 36 (2001) 2049.

[7] H.T. Philipp, L.J. Koerner, M.S. Hromalik, M.W. Tate and S.M. Gruner, Pixel array detector for the capture of femtosecond duration x-ray images, Proc. SPIE 6703 (2007) 670300.

[8] M.S. Hromalik, H.T. Philipp, L.J. Koerner, M.W. Tate and S.M. Gruner, Data acquisition and control for the LCLS pixel array detector, IEEE Nucl. Sci. Symp. Conf. Rec. 3 (2007) 1744.

[9] C.-T. Sah, J.Y.-C. Sun and J.J.-T. Tzou, Generation annealing kinetics of interface states on oxidized silicon activated by 10.2-ev photohole injection, J. Appl. Phys. 53 (1982) 8886.

[10] G.J. Hu and W.C. Johnson, Relationship between $x$-ray-produced holes and interface states in metal-oxide-semiconductor capacitors, J. Appl. Phys. 54 (1983) 1441.

[11] O. Evrard, M. Keters, L.V. Buul and P. Burger, A novel photodiode array structure for gamma camera applications, Nucl. Instrum. Meth. A 504 (2003) 188. 